\title{
Auswirkungen einer systematischen Patientenschulung in Hautpflege und -schutz in einer Handekzem-Spezialklinik
}

\section{Schlüsselwörter}

Handekzem · Präventionsprogramm · Hautpflege · Schutz ·

Patientenschulung

\section{Zusammenfassung}

Hintergrund: Ein Handekzem hat erheblichen Einfluss auf die Lebensqualität der betroffenen Person. Die Behandlung zielt vor allem darauf ab, die Funktion der Hautbarriere zu verbessern.

Ziele: Beurteilung der Wirkung und Akzeptanz eines neuartigen Schulungsprogramms für Handekzem-Patienten.

Methoden: Die Daten von 36 Patienten, die das Präventionsprogramm und eine Nachuntersuchung absolvierten, wurden retrospektiv analysiert. Dabei wurden der PGA-Score (PGA = physician global assessment), die Akzeptanz sowie Verhaltensänderungen bei den Teilnehmern beurteilt.
Ergebnisse: Bei $67 \%$ der Patienten trat eine Besserung des Handekzems ein, die der Wirkung unseres Schulungsprogramms zugeschrieben werden konnte. Der mittlere PGA-Score ging von 3 vor der Patientenschulung auf 2,2 bei der Nachuntersuchung signifikant zurück. Verhaltensänderungen im Hinblick auf Hautpflege und -schutz waren bei $81 \%$ bzw. $86 \%$ zu verzeichnen.

Schlussfolgerungen: Unser Schulungsprogramm hat positive Auswirkungen auf den klinischen Verlauf sowie auf die Adhärenz in Bezug auf Hautpflege und -schutz gezeigt. Die Einbindung in die Abläufe einer Handekzem-Klinik erwies sich als praktikabel, und das Programm stieß auf hohe Akzeptanz bei den Patienten.

(c) 2015 The Author(s). Published by S. Karger GmbH, Freiburg

Originalartikel

\section{Effects of Systematic Patient Education in Skin Care and Protection in a Hand Eczema Clinic}

Michela A.M. Corti Regina Stirnimann Luca Borradori Dagmar Simon

Universitätsklinik für Dermatologie, Inselspital, Universitätsspital Bern, Universität Bern, Bern, Schweiz his article is licensed under the Creative Commons Attribution-NonCommercial-NoDerivatives 4.0 International License (CC BY-NC-ND) distribution for commercial purposes as well as an distige and modified material requires written permission. 
Dr. phil. Ina Zschocke

ina.zschocke@sciderm.com

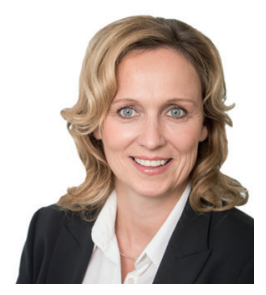

\section{Transfer in die Praxis}

Das Handekzem hat mit einer kumulativen Prävalenz von 15\%, einem chronischen Verlauf bei über 25\% der Patienten und einer Abheilungsrate von nur 28-40\% eine hohe medizinische Relevanz. Die indirekten Kosten der Erkrankung sind beträchtlich: Zahlen belegen, dass etwa ein Drittel der Patienten den Beruf wechseln müssen und ein Viertel - insbesondere ältere Patienten - ihre Arbeitsstelle krankheitsbedingt verlieren.

Bei der Behandlung des Handekzems spielen Hautpflege und -schutz im Zusammenhang mit der Wiederherstellung der Hautbarriere eine vorrangige Rolle. Ein Schulungsprogramm, das zu diesem Zweck an der Universitätsklinik Bern entwickelt wurde, ist anhand von 36 Patienten evaluiert worden. Ergänzend zur diagnostischen Abklärung und ärztlichen Behandlung wurden über geschultes Klinikpersonal (specialized nurse) Informationen zu Hautpflege und -schutz vermittelt sowie praktische Aspekte in Bezug auf den Arbeitsplatz und das heimische Wohnumfeld besprochen und trainiert. Informations- und Anwendungsmaterialien wurde zur Verfügung gestellt. Der Schulungsaufwand betrug 15-30 min pro Patient. Die Ergebnisse der Studie von Corti et al. belegen, dass gezielte Informationsvermittlung und praktische Übungen die Hautsymptomatik zusätzlich zu einer Arzneimittelbehandlung positiv beeinflussen. Die Patienten zeigten Änderungen auf der Verhaltensebene in Form von sinnvollem Hautschutz (Anwendung eines Schutzschaums / einer Schutzcreme oder Tragen von Handschuhen) und Hautpflege.

In der dermatologischen Behandlung haben sich in den letzten Jahren Schulungsprogramme für verschiedene Indikationen etabliert, die auf ein fundiertes Krankheitsverständnis des Patienten, Verhaltensaspekte und Adhärenz zur laufenden
Therapie abzielen. Der positive Einfluss dieser Schulungsprogramme auf den Krankheitsverlauf wie auch auf die Lebensqualität ist eindrucksvoll nachgewiesen worden; allen voran sind hier die von Ring et al. [1] entwickelten NeurodermitisTrainingseinheiten zu nennen.

Die kritischen Punkte entsprechender Schulungsprogramme sind oftmals deren mangelnde Praktikabilität im dermatologischen Praxisbetrieb oder in der Krankenhausroutine sowie die Akzeptanz der Patienten. Wird die Schulung zu komplex oder für den behandelnden Dermatologen zu zeitaufwändig, kann sie nicht erfolgreich eingeführt werden. Im vorliegenden Fall der Schulung für Patienten mit Handekzem ist dieser Aspekt gut gelöst: Mit dem 15-30-minütigen Schulungsaufwand pro Patient im Laufe der Behandlung durch spezialisiertes Personal kann der behandelnde Arzt sogar noch entlastet werden. Die positiven Rückmeldungen in der Studie, denen zufolge 92\% der Patienten der Ansicht waren, von dem Schulungsprogramm profitiert zu haben, unterstreichen zusätzlich dessen Wert. Sofern das entsprechende Informationsmaterial von der Universitätsklinik Bern zur Verfügung gestellt werden kann, sollte die Einführung der Schulung in der Therapie des Handekzems reibungslos möglich sein.

\section{Referenzen}

1 Ring J, Wahn U, Gieler U: Neurodermitisschulung: Ein neues Behandlungsprogramm zur sekundären Krankheitsprävention. Dtsch Arztebl 2001;98:A3202-A3209. www.aerzteblatt.de/archiv/29662/Neurodermitisschulung-Ein-neues-Behandlungsprogramm-zur-sekundaeren-Krankheitspraevention (letzter Abruf 16. März 2015).

Kontaktadresse: Dr. phil. Ina Zschocke, SClderm GmbH, Drehbahn 1-3, 20354 Hamburg, Deutschland 